\title{
Neurotoxic and Cardiotoxic Effects of Cocaine and Ethanol
}

\author{
Muhammad U. Farooq, MDa, Archit Bhatt, MD, MPHa, Mehul B. Patel, $M D^{b}$
}

aDepartment of Neurology and Ophthalmology, Michigan State University, East Lansing, MI

bDepartment of Cardiology, Michigan State University/Sparrow Health System, Lansing, MI

\begin{abstract}
Introduction: Concurrent abuse of alcohol and cocaine results in the formation of cocaethylene, a powerful cocaine metabolite. Cocaethylene potentiates the direct cardiotoxic and indirect neurotoxic effects of cocaine or alcohol alone.

Case Report: A 44-year-old female with history of cocaine and alcohol abuse presented with massive stroke in the emergency department. CT scan revealed extensive left internal carotid artery dissection extending into the left middle and anterior cerebral arteries resulting in a massive left hemispheric infarct, requiring urgent decompressive craniectomy. The patient had a stormy hospital course with multiple episodes of torsades de pointes in the first 4 days requiring aggressive management. She survived all events and was discharged to a nursing home with residual right hemiplegia and aphasia.

Conclusion: The combination of ethanol and cocaine has been associated with a significant increase in the incidence of neurological and cardiac emergencies including cerebral infarction, intracranial hemorrhage, myocardial infarction, cardiomyopathy, and cardiac arrhythmias. The alteration of cocaine pharmacokinetics and the formation of cocaethylene have been implicated, at least partially, in the increased toxicity of this drug combination.
\end{abstract}

\section{INTRODUCTION}

Cocaine mixed with alcohol is one of the most common recreational drug combinations in the US [1]. This combination results in longerlasting euphoric effects, with potentially lethal neurovascular or cardiovascular complications. The synergism is likely multifactorial due to enhanced cocaine absorption, inhibition of cocaine elimination, and formation of active metabolites like cocaethylene [2].

\section{CASE REPORT}

We describe the case of a 44-year-old female with history of cocaine and alcohol abuse who developed devastating neurological and cardiovascular complications. She had a history of hypertension that was fairly controlled by medications. She was brought to the emergency department in an unresponsive state with an endotracheal tube placed at the scene by the EMS. The initial examination revealed a regular pulse of $62-\mathrm{bpm}$, blood pressure of $187 / 99 \mathrm{mmHg}$, respiratory rate of $22 / \mathrm{min}$, and temperature of $99.1^{\circ} \mathrm{F}$. She was unresponsive to verbal requests and had minimal response to painful stimuli. She had dense right hemiplegia with left gaze preference. Her pupils were equal in size and minimally reactive to light bilaterally. She had right-sided upper motor neuron facial palsy and an impaired gag reflex. The deep tendon reflexes were brisk on the right side with bilaterally positive Babinskis.

The results of initial lab investigations, including complete blood count and blood biochemistry, are listed in the Table 1 . Her urine and blood toxicology screen was positive for cocaine and alcohol metabolites with blood alcohol level of $0.20 \mathrm{~g} / \mathrm{dl}$. Toxicology

Keywords: cocaine, alcohol, cocaethylene, neurotoxicity, cardiotoxicity

Notes: There was no outside funding of any kind used for this study.

Corresponding Author: Mehul B. Patel, MD, Department of Cardiology, Sparrow Health System, 1215 E. Michigan Avenue, Lansing, MI 48912.Email: mehulkhyati@gmail.com 


\section{Table 1: Initial Lab Values for Case Report}

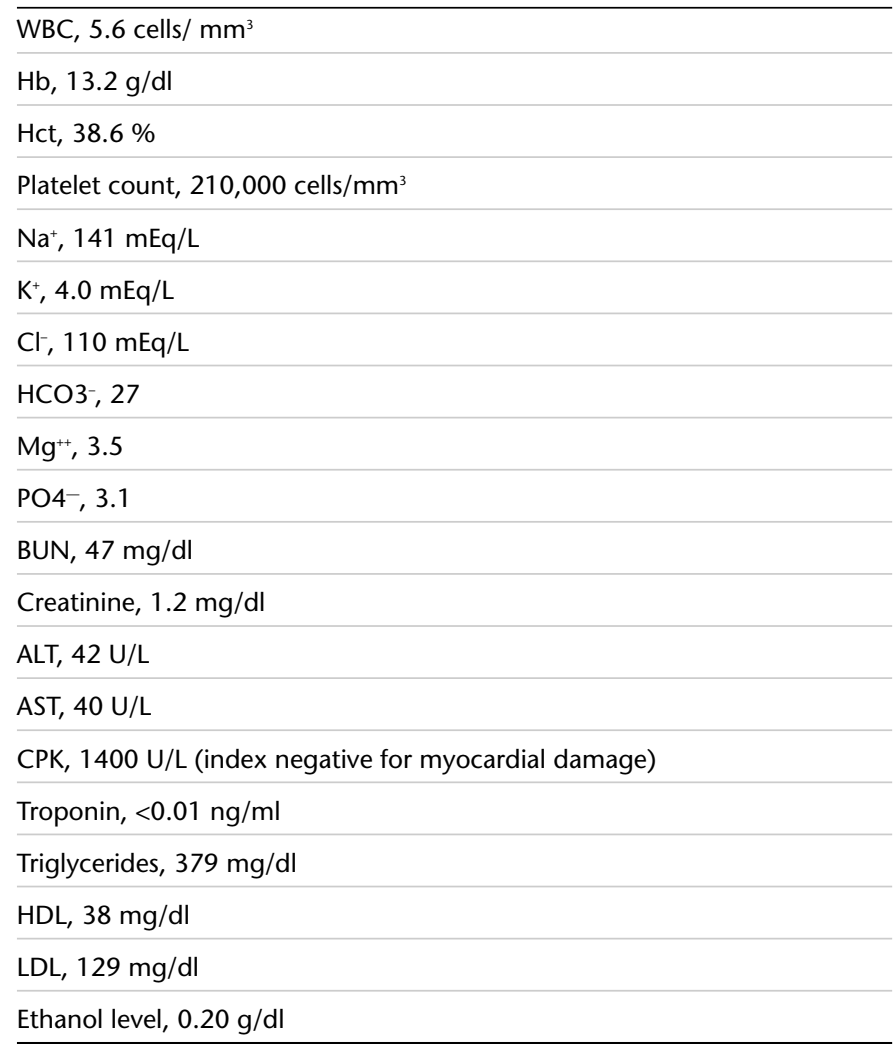

screening for opiates, phencyclidine, tricyclic antidepressants, amphetamines, methamphetamines, tetrahydrocannabinol, barbiturates, and benzodiazepine were all negative. The initial computerized tomography (CT) of the brain showed some early changes of cerebral infarction, including loss of the insular ribbon and hemispheric sulci effacement (Figure 1A). Magnetic resonance imaging (MRI) of the brain showed massive left cerebral hemispheric infarct (Figure $1 B$ and $C$ ) in the distribution of the anterior and middle cerebral arteries. Magnetic resonance angiography (MRA) of the head and neck showed dissection of the left internal carotid artery, and the internal carotid artery dissection extended into the left anterior and middle cerebral arteries, resulting in complete occlusion of these branches (Figure 1D). Transesophageal echocardiography (TEE) showed normal left ventricular ejection fraction (56\%) with no obvious source of embolus.

The patient was admitted at the stroke unit, with close continuous monitoring of neurological status. Her systolic blood pressure was controlled and maintained at $\geq 160 \mathrm{mmHg}$. She did not receive tissue plasminogen activator (t-PA), as the exact time of onset of her symptoms was not clear and moreover there was a significant risk of hemorrhage with t-PA therapy due to the massive cerebral infarction. She received $300 \mathrm{mg}$ of aspirin rectally every day. Day 2 showed a significant decline in the neurological status with repeat CT of the brain showing extensive peri-

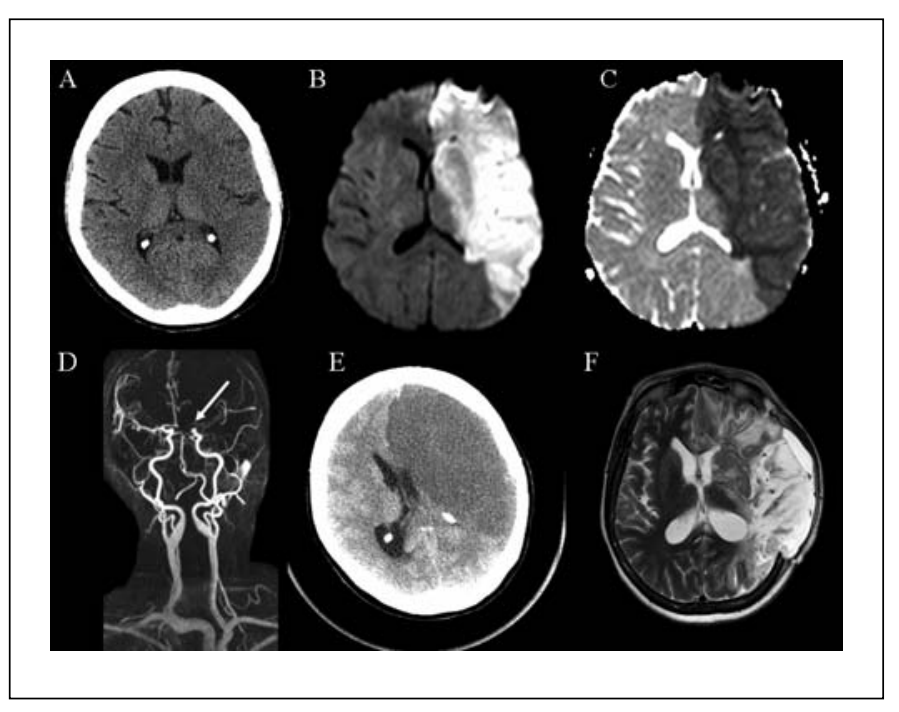

Figure 1: (A) CT of the brain with early changes of cerebral infarction without any midline shift. (B) Massive left hemispheric infarct on diffusion weighted image (DWI). (C) The corresponding infracted area on apparent diffusion coefficient (ADC) mapping. (D) MRA showing blockage of left internal carotid artery before its bifurcation into anterior and middle cerebral arteries. (E) CT of the brain showing midline shift due to worsening edema. (F) MRI brain showing hemicraniectomy defect in skull.

infarction edema and midline shift, as shown in Figure 1E. She received mannitol $(1 \mathrm{mg} / \mathrm{kg}$ ) along with $3 \%$ saline while she was emergently wheeled to the operating room where she underwent a left hemi craniectomy (Figure 1F). She tolerated the procedure well and had an uneventful outcome.

Her electrocardiogram (ECG) at the time of presentation showed a corrected QT interval (QTc) of $657 \mathrm{msec}$ (Figure 2A). While in the emergency department (ED), she had multiple episodes of torsades de pointes (Figure $2 B$ ). While most of these episodes were self-terminating, 2 episodes degenerated to ventricular fibrillation requiring emergency direct current defibrillation. The total number of episodes of torsades reduced consistently over time, and after 4 days of intravenous magnesium sulfate therapy her QT interval was $447 \mathrm{msec}$ with no further episodes of torsades (Figure 2C). She remained arrhythmia-free thereafter for the entire hospital stay of 34 days.

She survived the stormy and challenging hospital course requiring percutaneous bronchoscopically-guided tracheostomy and percutaneous endoscopically-guided gastrostomy tube placement. She was ultimately discharged to a nursing home with a normal QTc of $400 \mathrm{msec}$. Her modified Rankin Scale (mRS) at the time of discharge was 4 . She was admitted again for the elective duraplasty and bone flap replacement after 3 months. She continued to have dense right hemiplegia and aphasia without any significant recovery, with the same mRS of 4 . Her ECG revealed normal QT interval with no episodes of arrhythmia at the time of her second hospitalization for duraplasty. 


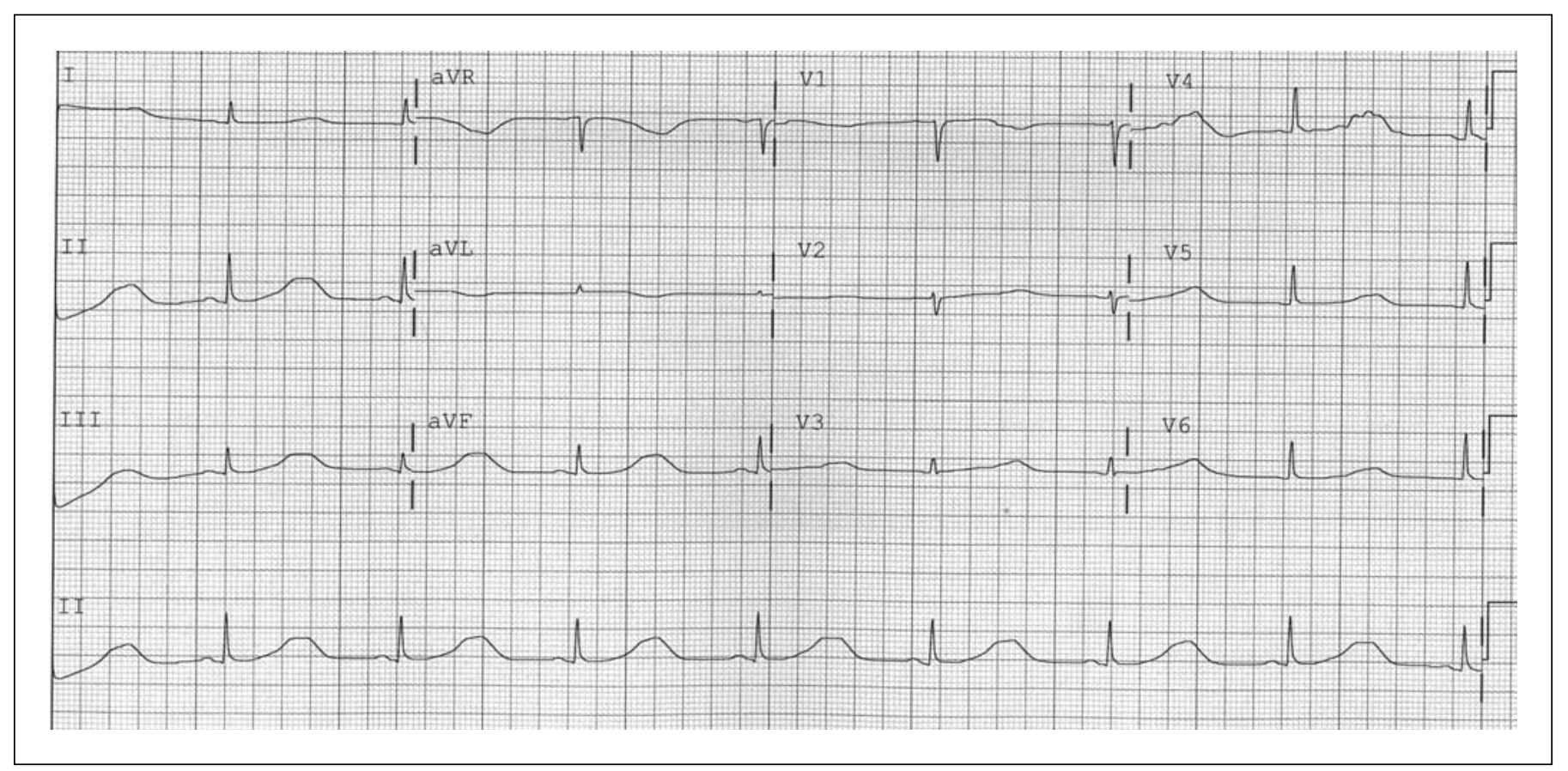

Figure 2: (A) Markedly prolonged QT interval (657 msec) at the time of hospitalization.

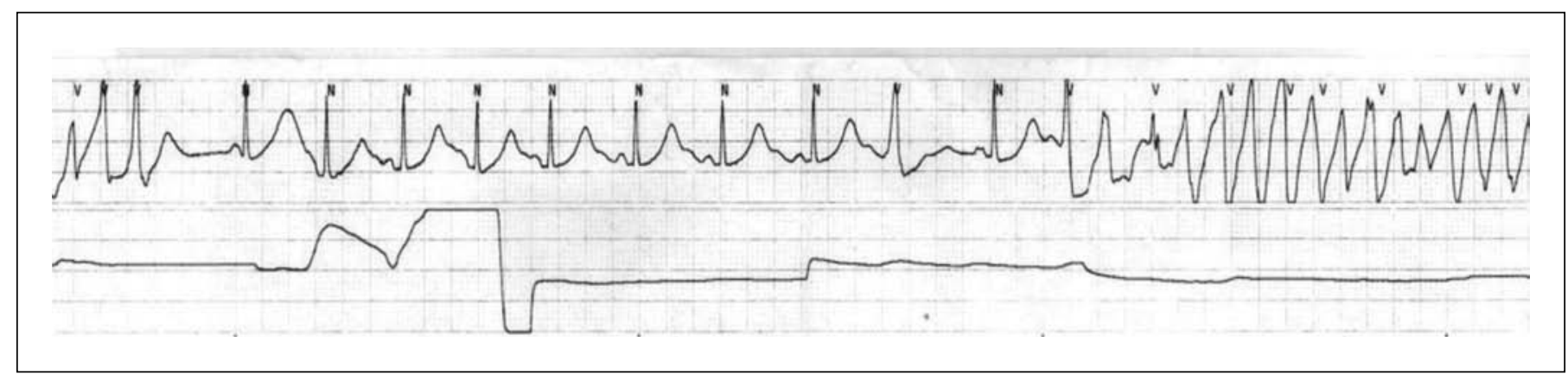

(B) An episode of torsades de pointes.

\section{DISCUSSION}

This case highlights the complications resulting from simultaneous intake of cocaine and ethanol. Cocaine is metabolized by hydrolysis of its ester groups. Benzoylecgonine, produced by demethylation of cocaine, is the major urinary metabolite and can be found in the urine for 2 to 5 days after a binge. Cocaine is frequently used in combination with other drugs that can modify its metabolism. Cocaine gets transesterified in the presence of ethyl alcohol to its ethyl homologue, cocaethylene. In vitro binding studies demonstrate the pharmacological profile of cocaethylene to be identical to that of cocaine at monoamine transport binding sites in the human brain. It is equipotent in binding with the dopamine receptors in the brain as well. The enhanced blockade of dopamine reuptake in the synaptic cleft by cocaethylene is likely to cause enhanced euphoria associated with combined alcohol and cocaine use [3]. This may explain the combined use of cocaine with ethanol by regular users; the Epidemiological Catchment Area data demonstrated that $85 \%$ of those who use cocaine concomitantly use alcohol [1].

The exact mechanism of cocaine toxicity resulting in vascular toxicity and neurotoxicity is not clear and is likely due to multiple factors. The potential mechanisms of its neurotoxicity include vasospasm, vasculitis, enhanced platelet aggregation, cardioembolism, and disruption of cerebral autoregulation [4]. It causes vasoconstriction due to its potent sympathomimetic properties. It prevents the reuptake of noradrenaline, serotonin, and dopamine at presynaptic nerve terminals and increases the release of calcium from the sarcoplasmic reticulum in cerebral vascular smooth muscle cells, resulting in vasoconstriction $[5,6]$. Endothelin-1 is another important mediator of cocaine-induced cerebral vasospasm [7]. Cocaine can cause cerebral vascular thrombosis and advanced ather- 


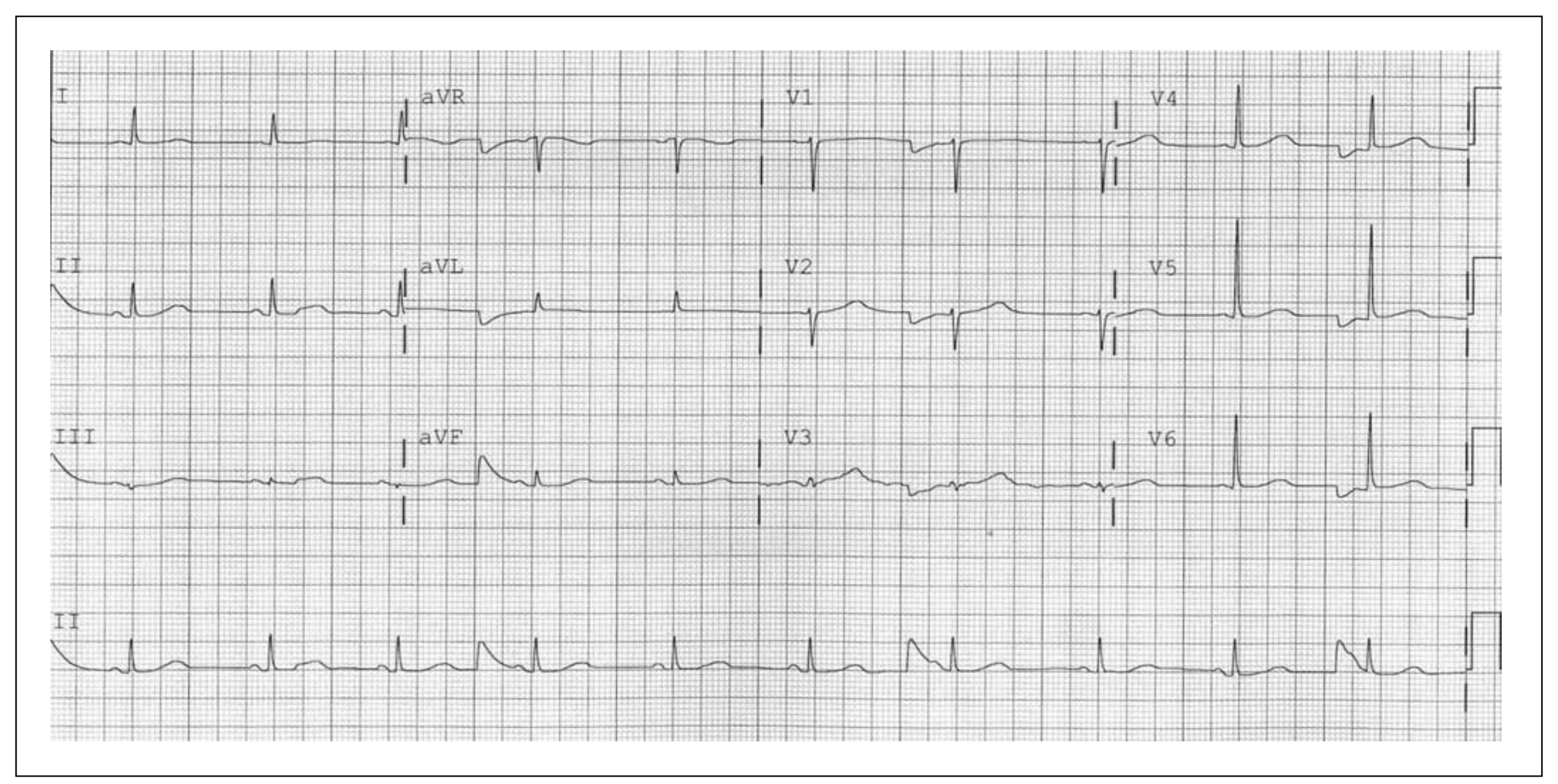

(C) Normal QT interval on day 4.

osclerosis of intracranial vessels. It increases thromboxane production and platelet aggregation, resulting in fatal cerebral infarction due to the formation of acute platelet-rich thrombi [8]. Another mechanism is cerebral vasculitis, which can be seen on cerebral angiography as arterial beading [9]. However, arterial beading is a nonspecific sign and only indicates vascular injury. Cocaine can also result in cardioembolism due to its cardiotoxic effects, including myocardial infarction and cardiomyopathy [10].

Cocaine blocks potassium channels, resulting in prolongation of action potential duration (APD) and QT interval $[11,12]$. Cocaine toxicity has been associated with different cardiac arrhythmias, including torsades, but the combination of cocaine and ethanol is considered to be more arrhythmogenic than either substance alone $[13,14]$. Our observation of torsades in this patient with combined alcohol and cocaine use further supports this view. There are other potential causes besides cocaine that may have produced torsades in this patient. The most obvious cause is the high intracranial pressure (ICP) due to malignant stroke, producing cardiac arrhythmias. The association of cardiac arrhythmia and other cardiac complications in patients with different neurological problems has been well documented in the literature $[15,16]$. Other potential causes include medications and toxins resulting in prolongation of QT interval and torsades. In our patient the time profile of cocaine ingestion matched well with increased QT interval in contrast to the time profile of raised ICP to QT prolongation. This patient had multiple episodes of torsades in the $\mathrm{ED}$, at which time there was no significant edema and midline shift, indicating normal ICP. Most of the complications of malignant stroke due to increased ICP happen after 48-72 hours, when there is development of edema and midline shift with potential risk of brainstem herniation. Patients with $>50 \%$ middle cerebral artery involvement have a significant risk of developing brain edema, which can be life threatening if not treated in time with aggressive measures. The fatality rates of malignant cerebral infarcts is approximately $80 \%$ in adults [17]. No medical treatment has been proven effective for these patients except for decompressive hemicraniectomy. Decompressive hemicraniectomy, if performed within 48 hours of MCI, significantly reduces mortality in adults $18-60$ years of age $[17,18]$. In our patient, the family initially refused the option of decompressive hemicraniectomy but later agreed when her condition deteriorated after the development of midline shift. At this stage, our patient did not develop any episode of torsades, thus the most logical explanation of torsades in this case is cocaine and alcohol use, most likely due to cocaethylene, but its quantitative measurement was not done.

Cocaine-induced torsades usually responds to traditional therapies such as intravenous magnesium and/or electrical pacing, and should be very carefully monitored in a neurointensive care setting because of the high risk of cardiac complication in critically ill patients with different neurological problems.

Our case demonstrates that combined use of cocaine and alcohol may increase the risk for cardiac and neurological complications in an intensive care setting. Patients with co-ingestion should be carefully monitored on telemetry, and neurocritical care physicians should take special caution in patients with a history of cocaine abuse, especially in combination with alcohol.

The authors have no potential financial conflicts of interest to report. 


\section{REFERENCES}

1. Regier DA, Farmer ME, Rae DS, et al. Comorbidity of mental disorders with alcohol and other drug abuse. Results from the epidemiologic catchment area (ECA) study. JAMA 1990;264:2511-2518.

2. Farre M, de la Torre R, Llorente M, et al. Alcohol and cocaine interactions in humans. J Pharmacol Exp Ther 1993;266: 1364-1373.

3. Hearn WL, Flynn DD, Hime GW, et al. Cocaethylene: A unique cocaine metabolite displays high affinity for the dopamine transporter. J Neurochem 1991;56:698-701.

4. Treadwell SD, Robinson TG. Cocaine use and stroke. Postgrad Med J 2007;83:389-394.

5. He GQ, Zhang A, Altura BT, et al. Cocaine-induced cerebrovasospasm and its possible mechanism of action. J Pharmacol Exp Ther 1994;268:1532-1539.

6. Du C, Yu M, Volkow ND, et al. Cocaine increases the intracellular calcium concentration in brain independently of its cerebrovascular effects. J Neurosci 2006;26: 11522-11531.

7. Fandino J, Sherman JD, Zuccarello M, et al. Cocaineinduced endothelin-1-dependent spasm in rabbit basilar artery in vivo. J Cardiovasc Pharmacol 2003;41:158-161.

8. Kugelmass AD, Oda A, Monahan K, et al. Activation of human platelets by cocaine. Circulation 1993;88:876-883.

9. Kaye BR, Fainstat M. Cerebral vasculitis associated with cocaine abuse. JAMA 1987;258:2104-2106.
10. Petty GW, Brust JC, Tatemichi TK, et al. Embolic stroke after smoking "Crack" Cocaine. Stroke 1990;21:1632-1635.

11. Chakko S, Sepulveda S, Kessler KM, et al. Frequency and type of electrocardiographic abnormalities in cocaine abusers (electrocardiogram in cocaine abuse). Am J Cardiol 1994;74: 710-713.

12. Kimura S, Bassett AL, Xi H, et al. Early afterdepolarizations and triggered activity induced by cocaine. A possible mechanism of cocaine arrhythmogenesis. Circulation 1992;85:2227-2235.

13. Singh N, Singh HK, Singh PP, et al. Cocaine-induced torsades de pointes in idiopathic long q-t syndrome. Am J Ther 2001;8:299-302.

14. Wilson LD, Jeromin J, Garvey L, et al. Cocaine, ethanol, and cocaethylene cardiotoxity in an animal model of cocaine and ethanol abuse. Acad Emerg Med 2001;8:211-222.

15. Jespersen CM, Fischer Hansen J. Myocardial stress in patients with acute cerebrovascular events. Cardiology 2008;110:123-128.

16. Tatschl C, Stollberger C, Matz K, et al. Insular involvement is associated with qt prolongation: ECG abnormalities in patients with acute stroke. Cerebrovasc Dis 2006;21:47-53.

17. Vahedi K, Hofmeijer J, Juettler E, et al. Early decompressive surgery in malignant infarction of the middle cerebral artery: A pooled analysis of three randomised controlled trials. Lancet Neurol 2007;6:21-222.

18. Mayer SA. Hemicraniectomy: A second chance on life for patients with space-occupying MCA infarction. Stroke 2007;38:2410-2412. 